\title{
Ultrasonography and Doppler ultrasonography in the evaluation of intraosseous lesions of the jaws
}

\author{
Saima Tariq ${ }^{1 *}$, Altaf Hussain Chalkoo ${ }^{2}$, Rizwan Hamid ${ }^{3}$ \\ ${ }^{1,3}$ Postgraduate, ${ }^{2}$ Professor, Dept. of Oral Medicine \& Radiology, Government Dental College, Srinagar, India
}

*Corresponding Author: Saima Tariq

Email: saima.ashawari5@gmail.com

\begin{abstract}
Objectives: To evaluate the efficacy of ultrasonography and colour and power Doppler ultrasonography for diagnosis of intraosseous lesions of the jaws and to correlate the contents of the lesion with the histological findings.

Materials and Methods: 30 patients with intraosseous jaw lesions in the maxilla or mandible were included in the study. Ultrasonographic examinations were performed and, according to the ultrasonography findings, the jaw lesions were classified into three groups: cystic, semisolid and solid. Further, colour Doppler ultrasonographic examination was performed to evaluate blood flow in all patients following which surgical treatment was done. The correlation between ultrasonography and Doppler ultrasonography findings of the lesions and histological findings was investigated.

Results: Thirty intra-osseous lesions from twenty nine patients were evaluated in this study. Twenty two lesions were located in the mandible and eight were in the maxilla. Of the thirty lesions, the ultrasound examination was inconclusive in one lesion because of the thick cortical vestibular bone plate and for two lesions histopathology was not available.

Conclusions: Information regarding the content of intraosseous jaw lesions can be obtained using ultrasound before any surgical procedure. Further, vascularization of the lesion can be assessed via Doppler ultrasonography. Also, there was a correlation between the ultrasound findings and the definitive histological diagnosis.
\end{abstract}

Keywords: Intraosseous, Ultrasonography, Doppler ultrasonography, Histopathology.

\section{Introduction}

Embryologically, the jaws originate from ectomesenchyme. As a result of this Odontogenic cysts and tumors are a commonly occurring lesions in the jaws. ${ }^{1}$ Correct early diagnosis and treatment of these lesions is necessary as some of these lesions show aggressive behaviour and even neoplastic transformation. ${ }^{2}$ Because of similar radiographic appearance diagnosing these Intraosseous jaw lesions often becomes confusing. ${ }^{3}$ Imaging, thereby, plays an important role in the diagnosis of these lesions. Various imaging modalities include radiography, computed tomography, magnetic resonance imaging, ultrasonography with Nuclear Medicine, Positron Emitting Tomography (PET), Singlephoton emission computed tomography (SPECT) among advanced modalities. Although computed tomography (CT) is of immense importance in diagnosing bone lesions but the radiation hazard and high cost limits its use. ${ }^{4}$

Ultrasonic imaging is one of the most important, developing diagnostic tools today. State-of-the-art ultrasonic scanners offer real-time gray scale images of anatomical details with millimeter spatial resolution superimposed on which a map of Doppler blood flow information is displayed in full color. ${ }^{5}$ Despite being use in medicine for years ultrasonography in dentistry is used mostly for soft tissue lesions. ${ }^{6}$ It is useful in differentiating soft-tissue tumors, cysts, salivary stones, relationship of swelling to adjacent structures, consistency whether solid or cystic, and also it is extremely suitable for follow-up examination of tumor patients. ${ }^{7}$ Diagnosing Intraosseous jaw lesions using ultrasonography usually becomes difficult due to overlying cortical bone thereby limiting its use, but the thin cortical bone in case of well grown and expanding lesions makes ultrasonograhy useful in diagnosis of such lesions. USG helps in differentiating solid and cystic components of the Intraosseous jaw lesions and in determining the site of biopsy. ${ }^{8}$ Improvements in the ultrasound technology and the introduction of high resolution ultrasonography have led to routine use of USG in examination and diagnosis of bone pathology. ${ }^{9}$

The use of color Doppler in ultrasound allows evaluation of the presence, nature and velocity of blood flow in ultrasound images of the examined tissue. It can also reveal the progressive formation of new vessels in bone during the initial healing period. ${ }^{10}$

\section{Aims \& Objectives of the Study}

1 To evaluate the ability of ultrasonography and Doppler ultrasonography for diagnosing Intraosseous jaw lesions.

2 To determine a correlation between ultrasonographic findings regarding the contents of the lesion with the histological findings.

\section{Study design}

After getting approval from the Institutional Ethics Committee, the study was conducted in the Department of Oral Medicine \& Radiology, Govt. Dental College, Srinagar over the period of a one and a half year commencing on $1^{\text {st }}$ June 2016.

\section{Materials and Methods}

1 A total of 30 patients with intraosseous jaw lesions based on their symptoms, clinical and panoramic radiographic findings were included in the study. 
2 An ultrasound examination was performed in all patients to detect the lesions and to evaluate their content, size, and the relationship with anatomical structures. All patients were informed about the study and signed an informed consent.

3 The patients then was done underwent Doppler ultrasound evaluation to evaluate blood flow within or surrounding the examined lesion by the same radiologist and the images were analysed at the same time the examination. The examinations were performed with a colour-coded duplex scanner (GE LOGIC S-8) with a 7-12 MHz linear-array transducer.

4 There was no histological result at the time of the examination and the radiologist did not have access to the radiographs before ultrasound examination.

5 The ultrasound probe was positioned outside the mouth on the skin overlying the intraosseous lesion. Its position was changed several times to obtain good quality transverse, oblique and longitudinal scans.

6 Histopathological examination of the lesions was done and the results were correlated with ultrasound examination.

\section{Inclusion criteria}

1. Patients of either gender presenting with intraosseous lesions in either maxilla or mandible.

\section{Results}

Thirty intra-osseous lesions from twenty nine patients were included in the study. Lesions were located mostly in the mandible (Twenty two) whereas maxilla had only eight lesions. Lesions mostly had a radiolucent appearance with well-defined borders on panoramic radiographs, except for two lesions. These lesions, histologically diagnosed as central giant cell granuloma \& round cell tumour, appeared mixed on panoramic radiography. Because of the thick cortical vestibular bone plate the ultrasound examination was inconclusive in one lesion and for two lesions histopathology was not available.

Table 1: Age distribution of study patients

\begin{tabular}{|l|c|c|}
\hline Age (years) & Frequency & Percentage \\
\hline$<20$ & 5 & 16.7 \\
\hline $20-34$ & 9 & 30.0 \\
\hline $35-49$ & 8 & 26.7 \\
\hline $50-64$ & 8 & 26.7 \\
\hline Total & 30 & 100 \\
\hline \multicolumn{2}{|c|}{ Mean \pm SD $=35.4 \pm 16.04$} \\
\hline
\end{tabular}

Table 2: Gender distribution of study patients

\begin{tabular}{|l|c|c|}
\hline Gender & Frequency & Percentage \\
\hline Male & 17 & 56.7 \\
\hline Female & 13 & 43.3 \\
\hline Total & 30 & 100 \\
\hline
\end{tabular}

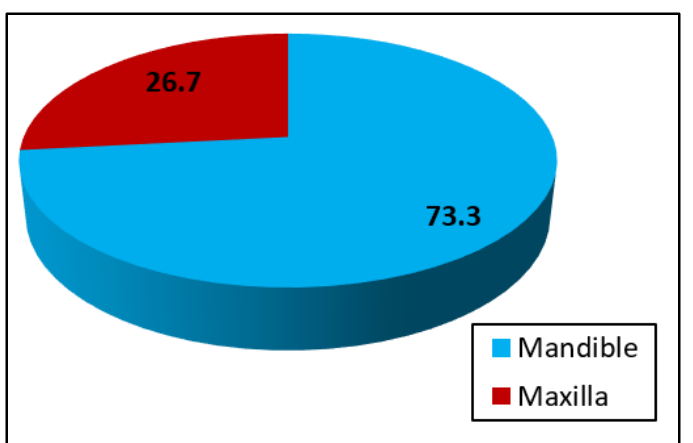

Fig. 1: Showing location of lesion on OPG in study patients

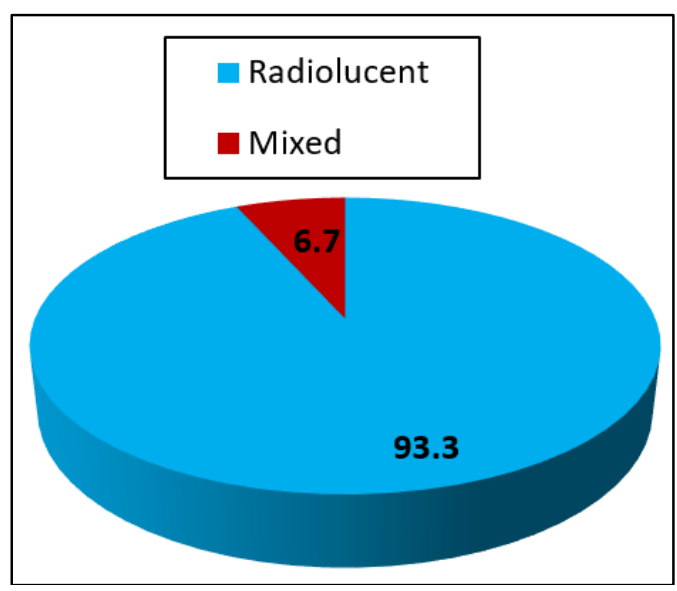

Fig. 2: Showing radiology of lesion in study patients

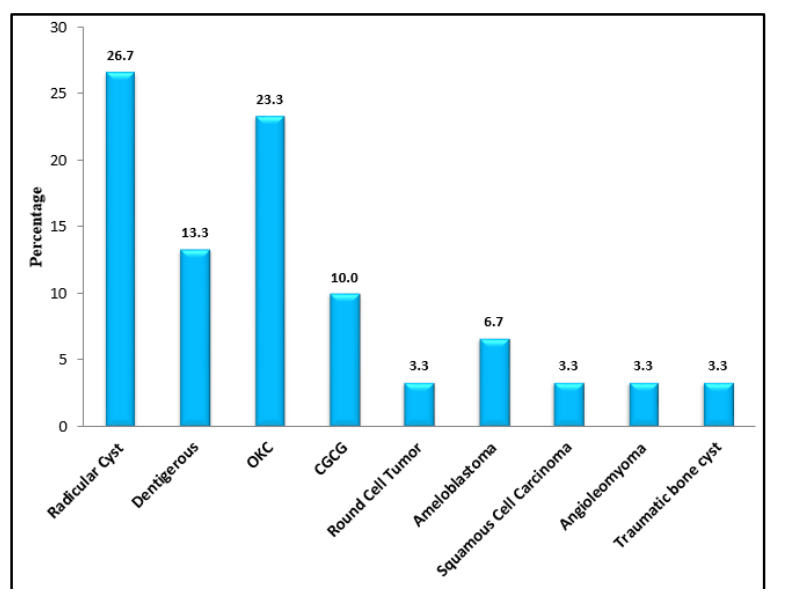

Fig. 3: Showing frequency of lesions according to histopathological diagnosis 
Table 3: The lesions ultrasound and Doppler findings and histological diagnosis

\begin{tabular}{|l|c|}
\hline Ultrasound and Doppler findings (n) & Histological diagnosis (n) \\
\hline Simple cyst- no evidence of vascularisation(2) & Radicular cyst (1) \\
& Traumatic bone cyst (1) \\
\hline $\begin{array}{l}\text { Simple Cyst with limited internal echoes-no evidence of } \\
\text { vascularisation(6) }\end{array}$ & Radicular Cyst (2) \\
& Residual cyst (1) \\
Dentigerous cyst (3)
\end{tabular}

CGCG, central giant cell granuloma; OSCC, Oral squamous cell carcinoma

Table 3 shows the ultrasound and Doppler findings along with histological diagnosis. Ultrasound examination showed a simple cystic appearance in 8 lesions, complex cystic appearance in 8 lesions, semisolid appearance in 9 lesions, solid appearance in 4 lesions of the 1 lesion, ultrasound examination was inconclusive due to thick vestibular bone plate. vascularisation was not detected on color Doppler ultrasound in 24 lesions.

Table 4: Showing correlation between histopathologic finding and US examination in cystic lesions

\begin{tabular}{|l|c|c|c|c|}
\hline Patient & Site & Radiology & $\begin{array}{c}\text { Ultrasonographic } \\
\text { Impression }\end{array}$ & Histopathological Findings \\
\hline 01 & Mandible & Radiolucent & Cystic & Dentigerous cyst \\
\hline 02 & Mandible & Radiolucent & Cystic & Traumatic bone cyst \\
\hline 03 & Maxilla & Radiolucent & Semisolid & Radicular cyst \\
\hline 04 & Mandible & Radiolucent & Cystic & Radicular cyst \\
\hline 05 & Maxilla & Radiolucent & Semisolid & Radicular cyst \\
\hline 06 & Mandible & Radiolucent & Semisolid & Dentigerous cyst \\
\hline 07 & Maxilla & Radiolucent & Cystic & Dentigerous cyst \\
\hline 08 & Mandible & Radiolucent & Cystic & Radicular cyst \\
\hline 09 & Mandible & Radiolucent & Semisolid & Radicular cyst \\
\hline 10 & Maxilla & Radiolucent & Cystic & Radicular cyst \\
\hline 11 & Mandible & Radiolucent & Cystic & Radicular cyst \\
\hline 12 & Mandible & Radiolucent & Cystic & Dentigerous cyst \\
\hline 13 & Mandible & Radiolucent & Semisolid & Radicular cyst \\
\hline
\end{tabular}

Table 4 shows the correlation between histopathologic findings \& ultrasound examination in cystic lesions. Of the 13 histologically confirmed cystic lesions ultrasound confirmed cystic lesions in $8(61.5 \%)$ cases semisolid appearance of remaining cystic lesions was attributed to these cysts being infected.

Table 5: Correlation between histopathologic finding and US examination in solid lesions

\begin{tabular}{|l|c|c|c|c|}
\hline Patient & Site & Radiology & $\begin{array}{c}\text { Ultrasonographic } \\
\text { impression }\end{array}$ & Histopathological findings \\
\hline 01 & Mandible & Mixed & Solid & Central giant cell granuloma \\
\hline 02 & Mandible & Radiolucent & Solid & Squamous cell carcinoma \\
\hline 03 & Maxilla & Radiolucent & Solid & Central giant cell granuloma \\
\hline 04 & Mandible & Radiolucent & Solid & Angioleomyoma \\
\hline 05 & Mandible & Radiolucent & Inconclusive & Ameloblastoma \\
\hline 06 & Maxilla & Mixed & Semisolid & Round cell tumor \\
\hline 07 & Mandible & Radiolucent & Semisolid & Central giant cell granuloma \\
\hline
\end{tabular}


Table 5 showing correlation between histopathologic finding and ultrasound examination in solid lesions out of 7 histologically confirmed solid lesions ultrasound showed solid lesions in 4(57.14\%) cases. For round cell tumour, usg showed areas of cystic degeneration within solid lesion hence semisolid impression was obtained. In case of central giant cell granuloma, the lesion was infected hence appeared semisolid on ultrasound. For ameloblastoma usg was inconclusive because of thick cortical bone plate.

Table 6: Correlation between histopathologic finding and US examination in mixed lesions

\begin{tabular}{|c|c|c|c|c|}
\hline Patient & Site & Radiology & Ultrasonographic impression & Histopathological findings \\
\hline 01 & Maxilla & Radiolucent & Semisolid & No HPE available \\
\hline 02 & Mandible & Radiolucent & Semisolid & Ameloblastoma \\
\hline 03 & Maxilla & Radiolucent & Semisolid & No HPE Available \\
\hline
\end{tabular}

Table 6 shows the correlation between histopathological findings and ultrasound examination. Histologically only one lesion diagnosed as ameloblastoma was found to be semisolid on usg for 2 lesions HPE was not available, hence correlation could not be ascertained.

Table 7: Correlation between histopathologic finding and US examination in keratocysts

\begin{tabular}{|c|c|c|c|c|}
\hline Patient & Site & Radiology & Ultrasonographic impression & Histopathological finding \\
\hline 01 & Mandible & Radiolucent & Cystic; thick viscid & Keratocyst \\
\hline 02 & Mandible & Radiolucent & Cystic; thick viscid & Keratocyst \\
\hline 03 & Mandible & Radiolucent & Cystic; thick viscid & Keratocyst \\
\hline 04 & Mandible & Radiolucent & Cystic; thick viscid & Keratocyst \\
\hline 06 & Mandible & Radiolucent & Cystic; thick viscid & Keratocyst \\
\hline 07 & Mandible & Radiolucent & Cystic; thick viscid & Keratocyst \\
\hline 08 & Mandible & Radiolucent & Cystic; thick viscid & Keratocyst \\
\hline
\end{tabular}

Table 7 shows the correlation between histopathologic findings and ultrasound examination in keratocysts out of 7 histologically diagnosed cases of keratocysts usg showed thick viscid cystic contents in all lesions giving a 100\% correlation.
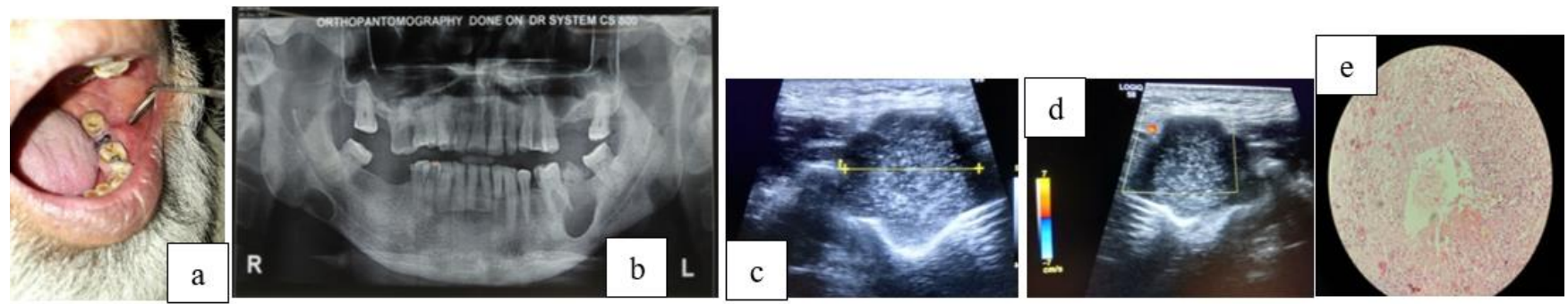

Fig. 1a: Intraoral pic showing vestibular obliteration IRT 36 37; b: OPG shows a well defined unilocular lesion associated with distal root of $36 ; \mathbf{c}, \mathbf{d}$ : USG showing hypoechoic area measuring $1.8 \times 1.5 \mathrm{~cm} \&$ color Doppler showing no vascularity within the lesion; e: HPE shows features of radicular cyst
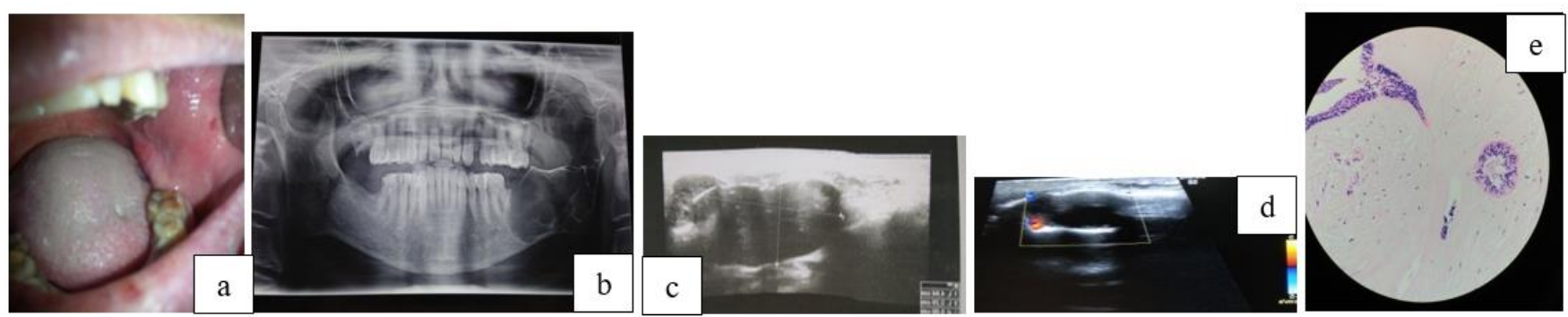

Fig 2a: Intraoral pic showing barely noticeable swelling of alveolar ridge IRT 38; b: OPG shows well defined multilocular radiolucent lesion involving mandibular angle and ramus; c, d: USG showing hypoechoic area measuring 3.1X4.4X2.3CMn\& color Doppler shows no vascularity within the lesion; e: HPE shows features of OKC 


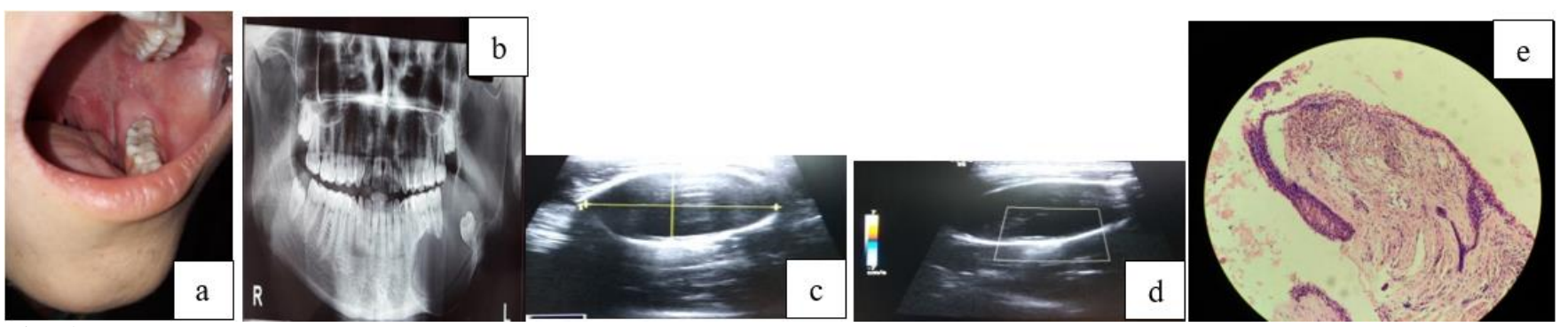

Fig. 3a: An expansile lesion present IRT 38; b: OPG showing a well defined multilocular radiolucent lesion reaching upto sigmoid notch of left ramus associated with an unerupted tooth; c: USG showing hypoechoic area measuring $3.5 \times 3.7 \mathrm{X} 2.2$ $\mathrm{cm}$; d: Color doppler shows no vascularity within the lesion; e: HPE shows features of dentigerous cyst

\section{Discussion}

Intraosseous lesions of jaws are commonly encountered lesions whose diagnosis poses a challenge owing to their similar clinical and radio graphic presentation. Conventional radiographs, although serve as primary diagnostic imaging modality but being two dimensional only limits their use. Moreover, they lack the ability to reveal the nature and content of the lesions. The cost and being not readily available limits the use of advanced imaging modalities like CT \& MRI. Among the advanced imaging modalities USG is an inexpensive, readily available and non hazardous modality that gives information not only about the location $\&$ size of lesion but also $\mathrm{p}$ about the contents of lesions. Further, color Doppler provides information about the vascularity of lesion that further aids in the diagnosis. Although ultrasound is indispensabily being used for soft tissue pathologies, very little has its use in diagnosing intraosseous lesions been explored.

US can also be used for diagnosis of many other cysts in the head and neck regions both hard and soft tissue cyst extending to hard tissues or soft tissues or vice versa. It can provide accurate information on the pathological nature of the lesion, content of lesion, inflammatory process, and capsular thickness of lesions. It can also differentiate between simple and complex cyst, and on nature of the content differentiate into solid and semisolid cysts. Simple cysts are totally anechoic with limited or without internal echoes, e.g. radicular cyst. Complex cysts have dense internal echoes or high echo, e.g. OKC. Solid lesions have a moderate echo. ${ }^{11}$

The present study evaluated the efficacy of ultrasound $\&$ color Doppler in diagnosing intraosseous jaw lesions. The study revealed that ultrasound along with color Doppler was able to show the contents \& vascularity of almost all intraosseous lesions except one lesion where because of thick cortical bone ultrasound was inconclusive. The study also showed a correlation was found between ultrasound findings and histological diagnosis. Among the Intraosseous lesions odontogenic cysts $(66.6 \%)$ were most commonly diagnosed.

The age of patients ranged from 6-64 years with a mean age of $35.4 \pm 16.04$ years. In a study conducted by Benjamin et $a{ }^{12}{ }^{12}$ similar age range of patients 4-64 years was observed. In this study seventeen $(56.7 \%)$ lesions were in males and thirteen $43.3 \%$ ) in females with a M:F ratio of 1.3. This is in accordance with the study conducted by Benjamin Fomete et al, ${ }^{12}$ where thirty-five $(54.7 \%)$ lesions were in males and twenty- nine $(45.3 \%)$ in females giving a $\mathrm{M}: \mathrm{F}$ ratio of 1.2:1. The greater frequency in adult males may be because they are more likely to neglect their teeth or they are more likely to sustain trauma to their teeth, compared to females, all of which may be the etiology for cyst formation

The lesions were more common in mandible, twenty two $(73.3 \%)$, than maxilla, eight $(26.7 \%)$. This is in accordance with the study conducted by Fabio et al, ${ }^{13}$ where the mandible was affected more frequently $(71.43 \%)$ than the maxilla (28.57\%) and Kambalimath et $\mathrm{al}^{14}$ where they found that the most common locations of the odontogenic cysts were the mandibular $(49.33 \%)$ and posterior region $(33.33 \%)$. A study by Sumer et al ${ }^{15}$ revealed that $50 \%$ of lesions were in mandible and $50 \%$ in maxilla.

Twenty eight $(93.34 \%)$ lesions had radiolucent appearance and two (6.66\%) had mixed appearance on panoramic radiograph. Study by Javadian Langaroodi A et $\mathrm{al},{ }^{16}$ reported that cysts and benign tumors had a radiolucent appearance as opposed to tumor-like lesions with a dominant radiopaque presentation. Cysts and benign tumors were well-defined with corticated borders, while tumor-like lesions were equally well-corticated and sclerotic.

In the study Odontogenic cyst was diagnosed by histological examination in nineteen $(63.3 \%)$ specimens. Rest of the lesions were diagnosed as CGCG three (10\%), ameloblastoma two $(6.6 \%)$, round cell tumour $1(3.3 \%)$,oral squamous cell carcinoma one (3.3\%), angioleomyoma one (3.3\%)and traumatic bone cyst one(3.3\%).This finding of our study is similar to Sumer et al study ${ }^{15}$ where seventeen (77.2\%) out of twenty- two specimens were diagnosed as odontogenic cysts.

Among the odontogenic cysts eight (26.7\%) were Radicular cysts, seven (23.3\%) were OKC, four (13.3\%) dentigerous cyst. A study by Jones et $\mathrm{al}^{17}$ showed that Radicular cyst was the most common diagnosis (52.3\%), followed by dentigerous cyst (18.1) and odontogenic keratocysts $(11.6 \%)$. A study by Manor et $\mathrm{a}^{18}$ showed that out of 322 patients with cystic lesions of the jaw $155(48 \%)$ were radicular cysts, 80 (25\%) were dentigerous cysts, 23 (7\%) were odontogenic keratocyst (=keratocystic odontogenic tumor), 19 (6\%) were eruption cysts, $16(5 \%)$ 
were traumatic bone cysts, and 29 (9\%) were nonodontogenic cysts. A study by Kambalimath et al ${ }^{14}$ showed that Radicular cyst was most prevalent histological type $(48.67 \%)$ followed by dentigerous cyst, odontogenic keratocyst, lateral periodontal cyst, paradental cyst, residual cyst, adult gingival cyst, glandular odontogenic cyst, calcifying odontogenic cyst. Study by Benjamin Fomete et $\mathrm{al}^{12}$ reported dentigerous cyst $(\mathrm{n}=21 ; 32.8 \%)$ was the most predominant lesion followed by periodontal $(\mathrm{n}=12 ; 18.8 \%)$ and radicular cysts $(\mathrm{n}=10 ; 15.6 \%)$. Study by Javadian Langaroodi A et al, ${ }^{16}$ reported $\mathrm{OKC}$ to be the most prevalent cyst. Our study is in partial agreement with above mentioned studies and in complete contrast to Benjamin Fomete et al. ${ }^{12}$

The size of the lesions ranged from $2.38 \mathrm{~cm}^{3}$ to 52.98 $\mathrm{cm}^{3}$ with a median of $21.51 \mathrm{~cm}^{3}$. A study by Gad et $\mathrm{al}^{19}$ showed that lesion sizes ranged from 0.019 to $40.856 \mathrm{~cm} 3$ with a median of $0.622 \mathrm{~cm} 3$. Most lesions $(71 \%)$ were smaller than $5 \mathrm{~cm}^{3}$. Ultrasound examination showed eight lesions as simple cysts. These lesions were anechoic to hypoechoic and had no vascularity on color Doppler. In all these cases, the findings of ultrasound and Doppler ultrasound were compatible with the content of the lesion. These findings are in accordance with Sumer et $\mathrm{al}^{15}$ where in case of simple cysts, USG findings were compatible with the content of the lesions. Study by Shahidi et $\mathrm{al}^{20}$ reported complete agreement between the USG and histopathologic results regarding content assessment

Cysts are usually anechoic due to their liquid content that produce no internal echoes.If the cyst becomes infected then the content of the lesion can produce echoes producing a hypoechoic picture as described by ishikawa,pogrel,osama\&corney. ${ }^{77}$

The sensitivity of ultrasound examination in case of cystic lesions was found to be $61.5 \%$.This implies that out of 13 histopathologically diagnosed cysts, ultrasound examination showed $8(61.5 \%)$ lesions to be cystic. Semisolid contents on ultrasound examination in the remaining 5 cystic cases can be explained on the basis of the associated inflammatory process seen in them on HPE. Study by Lauria et $\mathrm{al}^{21}$ showed that ultrasound examination was compatible with histological examination in $73.9 \%$ of cases in case of cystic lesions. Chandak et $\mathrm{al}^{22}$ reported that for cystic swellings sonographic diagnosis had a sensitivity of $100 \%$ and a specificity $98.3 \%$.

In the study complex cystic lesions on ultrasound examination showed dense hypoechoic cystic content with no evidence of vascularisation. These cystic lesions were mainly found to be odontogenic keratocysts. Studies by Sumer et $\mathrm{al}^{15}$ and Lauria et $\mathrm{al}^{21}$ also showed that okc had dense cystic content. Pallagi et $\mathrm{al}^{23}$ in their study found that okc exhibited hypoechoic pattern on usg examination.

In case of keratocysts USG examination was in complete agreement with A histopathological findings. out of 7 histologically diagnosed cases of okc, usg examination showed all of them to be complex cysts. In the study by Lauria et $\mathrm{al}^{21} 77.7 \%$ of cases were diagnosed as complex cystic on ultrasound examination.
In the study semisolid content was found on ultrasound examination in nine cases with vascularity in only one case on color Doppler. Histopathologically these lesions were diagnosed as Radicular cyst (4), dentigerous cyst(1) and ameloblastoma (1). For two lesions HPE was not available. All these lesions had no vascularity on color Doppler. One semisolid lesion diagnosed as Round cell tumour was vascular on color Doppler. Study by Sumer et al ${ }^{15}$ found 6 lesions to be semisolid with no evidence of vascularisation [Radicular 2, residual 2, dentigerous cyst 2]. In a study by Lauria et $\mathrm{al}^{21}$ mixed lesions were mainly found to be ameloblastoma on HPE.

Round cell tumour was seen as semisolid lesion on ultrasound examination. This may be attributed to cystic degeneration within solid component as seen on ultrasound examination. Ameloblastoma was seen to have semisolid content on ultrasound examination which may be attributed to its association with an impacted tooth. Ameloblastomas occur in association with an impacted or unerupted tooth (mostly the mandibular third molar). ${ }^{24}$ Thick cortical bone led to inconclusive ultrasound examination in one case only.

In the study solid content on ultrasound and vascularity on color doppler was found in four cases. These were diagnosed to be CGCG (2), OSCC (1) and angioleomyoma (1) respectively. Study by Sumer et $\mathrm{al}^{15}$ showed 2 solid lesions with internal vascularisation on ultrasound examination.

For solid lesions the sensitivity of ultrasound examination was $57.14 \%$ which implies that out of 7 histopathologically diagnosed solid cases, ultrasound examination showed only $4(57.14 \%)$ cases to be solid.

Ultrasound examination was inconclusive for one lesion that was diagnosed to be ameloblastoma on HPE. This was because of thick cortical bone plate. Studies by Sumer et al ${ }^{15}$ and Lauria et $\mathrm{al}^{21}$ also showed that ultrasound examination was inconclusive in some cases due to thick cortical bone plate.

\section{Conclusion}

The results of our study show that ultrasound along with color Doppler was able to reveal the contents and vascularity of the lesion with a definitive correlation between ultrasonographic findings \& histological diagnosis. Odontogenic cysts comprised the most common group of Intraosseous lesions. Ultrasonography showed most of the lesions to be hypoechoic and non vascular. Although the purpose of ultrasonography of intraosseous lesions is not to establish the definitive diagnosis, it will facilitate the differential diagnosis between solid and cystic lesions and is an excellent guide to biopsy in a more representative area.

\section{Source of funding \\ None.}

\section{Conflict of interest}

None. 


\section{References}

1. Slootweg PJ. Lesions of the jaws. Histopathol 2009;54:401-18.

2. Baghaei F, Zargaran M, Najmi HR, Moghimbeigi A. A clinicopathological study of odontogenic cysts and tumors in Hamadan, Iran. J Dent Shiraz Univ Med Sci 2014;5(4):167-72.

3. Raitz R, Correa L, Curi MM, Dib LL, Fenyo-Pereira M. Conventional and indirect digital radiographic interpretation of oral unilocular radiolucent lesions. Dentomaxillofac Radiol 2006;35:165-9.

4. Mallorie CNJ, Jones SD, Drage NA, Shepherd SD. The reliability of high resolution ultrasound in the identification of pus collection in head and neck swellings. Int J Oral Maxillofac Surg 2012;41:252-5.

5. Shung KK. High Frequency Ultrasonic Imaging. J Med Ultrasound 2009;17(1):25-30.

6. Imbeau J. Introduction to through-transmission alveolar ultrasonography (TAU) in dental medicine. Cranio 2005;23:100-12.

7. Mukhi PU, Mahindra UR. The use of ultrasonography in diagnosis and management of superficial facial space infections. Indian J Dent Res 2012;23:313-9.

8. Singh GP, Dogra S, Kumari E. Ultrasonography: maxillofacial applications. Ann Dent Specialty 2014;2(3).

9. Adeyemoa WL, Akadirib OA. A systematic review of the diagnostic role of ultrasonography in maxillofacial fractures. Int J Oral Maxillofac Surg 2011;40:655-61.

10. Tikku AP, Kumar S, Loomba K, Chandra A, Verma P, Aggarwal R. Use of ultrasound, color Doppler imaging and radiography to monitor periapical healing after endodontic surgery. J Oral Sci 2010;52(3):411-6.

11. Dharti N, Neerjesh P, Wadhawan R. Ultrasonography; A boon as a diagnostic \& therapeutic aid in dentistry: A review. IJBAR 2014;05(10).

12. Benjamin Fomete. A 10-year retrospective analysis of 64 cases of cystic lesions of the oral and maxillofacial Region in a Nigerian Tertiary Hospital. Oman Med J 2016;31(6):434-8.

13. Araujo JP, Lemos CA, Miniello TG, Alves FA. The relevance of clinical and radiographic features of jaw lesions: A prospective study. Braz Oral Res 2016;30(1):e96.

14. D. H. Kambalimath, H. V. Kambalimath, S. M. Agrawal. Prevalence and Distribution of Odontogenic Cyst in Indian Population: A 10 Year Retrospective Study. J Maxillofac Oral Surg 2014;13(1):10-5.
15. Sumer AP, Danaci M, Ozen Sandikçi E, Sumer M, Celenk P. Ultrasonography and Doppler ultrasonography in the evaluation of intraosseous lesions of the jaws. Dentomaxillofac Radiol 2009;38:23-7.

16. Javadian Langaroodi A. Intraosseous Benign Lesions of the Jaws: A Radiographic Study. Iran J Radiol 2014;11(1):e7683.

17. Jones AV, Craig GT, Franklin CD. Range and demographics of odontogenic cysts diagnosed in a UK population over a 30-year period. J Oral Pathol Med 2006;35:500-7.

18. Esther Manor, Leonid Kachko, Max B. Puterman, George Szabo, Lipa Bodner. Cystic Lesions of the Jaws - A Clinicopathological Study of 322 Cases and Review of the Literature. Int J Med Sci 2012;9(1):20-6.

19. Gad K, Ellabban M, Sciubba J. Utility of Transfacial Dental Ultrasonography in Evaluation of Cystic Jaw Lesions. $J$ Ultrasound Med 2018;37(3):635-44.

20. Shahidi SH, Shakibafard A, Zamiri B, Mokhtare MR, Houshyar M, Mahdian S. The Feasibility of Ultrasonography in Defining the Size of Jaw Osseous Lesions. Dent Shiraz Univ Med Sci 2015;16(4):335-40.

21. Lauria L, Curi MM, Chammas MC, Pinto DS, Torloni H. Ultrasonography evaluation of bone lesions of the jaw. Oral Surg Oral Med Oral Pathol Oral Radiol Endod 1996;82:351-7.

22. Chandak R, Degwekar S, Bhowte RR. An evaluation of efficacy of ultrasonography in the diagnosis of head and neck swellings. Dentomaxillofac Radiol 2011;40:213-221.

23. Pallagatti S, Sheikh S, Puri N, Mittal A, Singh B. To evaluate the efficacy of ultrasonography compared to clinical diagnosis, radiography and histopathological findings in the diagnosis of maxillofacial swellings. Eur J Radiol 2012;81:1821-7.

24. Hamed Mortazavi, Maryam Baharvand. Jaw lesions associated with impacted tooth: A radiographic diagnostic guide. Imaging Sci Dent 2016;46:147-57.

How to cite this article: Tariq S, Chalkoo AH, Hamid R. Ultrasonography and Doppler ultrasonography in the evaluation of intraosseous lesions of the jaws. J Oral Med, Oral Surg, Oral Pathol, Oral Radiol 2020;6(1):5-11. 